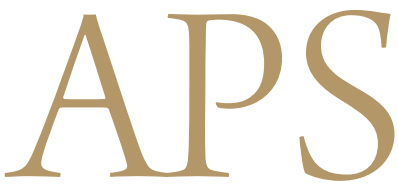

Archives of Plastic Surgery

\title{
When Are Circular Lesions Square? A National Clinical Education Skin Lesion Audit and Study
}

\author{
Benjamin H Miranda, Katie A Herman, Marco Malahias, Ali Juma \\ Plastic and Reconstructive Surgery Department, Countess of Chester Hospital NHS Foundation Trust, Chester, UK
}

Background Skin cancer is the most prevalent cancer by organ type and referral accuracy is vital for diagnosis and management. The British Association of Dermatologists (BAD) and literature highlight the importance of accurate skin lesion examination, diagnosis and educationally-relevant studies.

Methods We undertook a review of the relevant literature, a national audit of skin lesion description standards and a study of speciality training influences on these descriptions. Questionnaires $(n=200)$, with pictures of a circular and an oval lesion, were distributed to UK dermatology/plastic surgery consultants and speciality trainees (ST), general practitioners (GP), and medical students (MS). The following variables were analysed against a pre-defined 95\% inclusion accuracy standard: site, shape, size, skin/colour, and presence of associated scars.

Results There were 250 lesion descriptions provided by 125 consultants, STs, GPs, and MSs. Inclusion accuracy was greatest for consultants over STs (80\% vs. 68\%; $P<0.001)$, GPs (57\%) and MSs $(46 \%)(P<0.0001)$, for STs over GPs $(P<0.010)$ and MSs $(P<0.0001)$ and for GPs over MSs $(P<0.010)$, all falling below audit standard. Size description accuracy sub-analysis according to circular/oval dimensions was as follows: consultants (94\%), GPs (80\%), STs (73\%), MSs (37\%), with the most common error implying a quadrilateral shape (66\%). Addressing $B A D$ guidelines and published requirements for more empirical performance data to improve teaching methods, we performed a national audit and studied skin lesion descriptions. To improve diagnostic and referral accuracy for patients, healthcare professionals must strive towards accuracy (a circle is not a square).

Conclusions We provide supportive evidence that increased speciality training improves this process and propose that greater focus is placed on such training early on during medical training, and maintained throughout clinical practice.

\section{Keywords Clinical audit / Skin neoplasms / Education / Study / Surgery, plastic}

\author{
Correspondence: \\ Benjamin H Miranda \\ Plastic and Reconstructive Surgery \\ Department, Countess of Chester \\ Hospital NHS Foundation Trust, \\ Countess of Chester Health Park, \\ Liverpool Road, Chester CH2 1UL, UK \\ Tel: +44-0-7961-996-229 \\ Fax: +44-0-20-8202-5778 \\ E-mail: DrBMiranda@googlemail.com
}

No potential conflict of interest relevant to this article was reported.

\section{INTRODUCTION}

In 2010, 324,579 new cancer cases (excluding non-melanoma skin cancer) were registered in the UK, with melanoma representing 3.9\% [1]. Additionally, 99,549 cases of non-melanoma skin cancer were also registered, a likely under-representation, as many are managed within the community [2]. Skin cancers therefore represent $25 \%$ of cancers diagnosed annually and are the commonest cancer by organ type. Furthermore 2,203 UK patients died from skin cancer in 2010, illustrating its signifi- 
cance for the general population [2].

The NHS Care Plan (2000) Cancer Management Goals, published following the UK government white paper on the same subject (1999) [3], include primary to secondary care referral within 24 hours, a '2-week wait' ruling for specialist consultation within 2 weeks of referral, and treatment within 62 days [4]. As professionals, we strive towards precision to achieve optimal results for patients. Evidence-based training helps us understand protocol whilst still retaining independent thought to tailor patient care. British Association of Dermatologists (BAD) undergraduate guidelines highlight the importance of recognising skin cancers $[5,6]$. However, despite the fact that cutaneous disease represents the greatest new primary care consultation workload (24\%), dermatological training receives under $1 \%$ of undergraduate teaching time $[7,8]$.

To inter-communicate effectively, referral terminology should be 'gold-standardised' just as it is in other areas of medicine: for example, the left atrium is exactly that, not the right, or the ventricle. General practitioners (GP) letters or proformas should facilitate efficient secondary care referral within the '2-week wait' ruling for melanoma [9]. In certain cases, such as referrals between secondary care services or GP referrals for potentially benign lesions, a letter is commonly utilised. It is therefore important that healthcare professionals describe lesions accurately. We are educated in systems that assist with this, such as 'textbook answer' lesion inspection protocols: site, shape, size, symmetry, skin colour and scarring [10]. Journal publications also present lesion description systems, such as $\mathrm{ABCDE}$ for malignant melanoma (asymmetry, border, colour, diameter, evolving features) [11-13]. Furthermore, the need for increased dermatological training and the requirement for more empirical performance data to improve teaching methods have been well

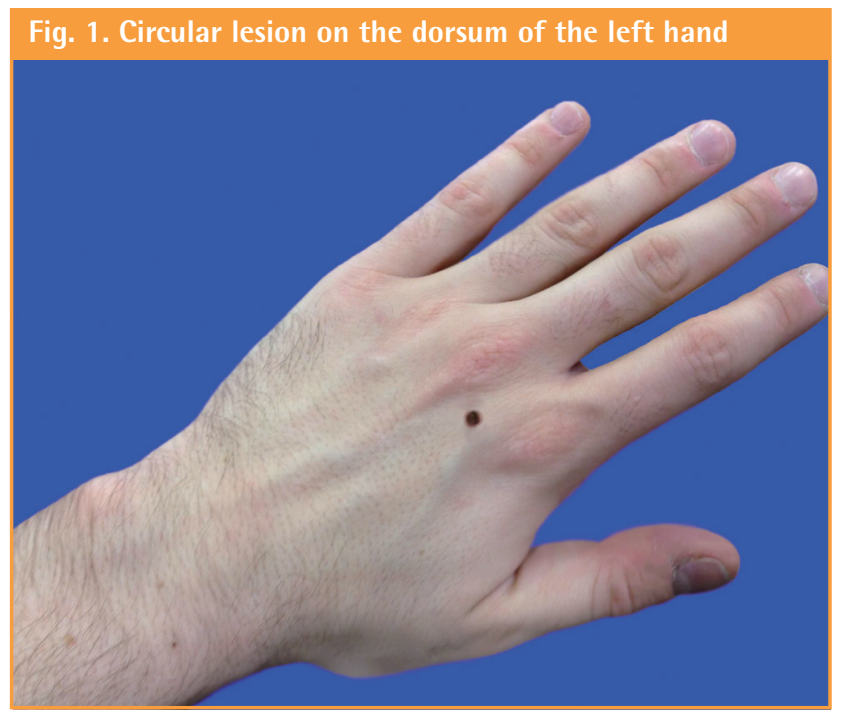

documented $[14,15]$.

In order to address the potential pitfalls in accurate referral and diagnosis of skin lesions, particularly in the context of widespread sun damage, and to address calls in the literature for further studies on education and training to ultimately improve clinical practice, we performed a national audit of skin lesion description standards by consultant dermatologists and plastic surgeons, GP, dermatology and plastic surgery registrar speciality trainees (ST) and medical students (MS); we also studied the effects of speciality training on their descriptions. The null hypotheses were that audit standards would be met by qualified doctors, that a difference would be demonstrated between medical students and qualified doctors, with no differences demonstrated between medical students and qualified doctors.

\section{METHODS}

After clinical governance approval, an audit and study of dermatology and plastic surgery consultants and STs, GPs and MSs was undertaken during 2012. Two hundred, 3-question questionnaires were nationally distributed across 16 UK NHS Deaneries. Question 1 requested the participant's training grade/job title and questions 2 and 3 requested 2 lesion descriptions (Fig. 1). Answers were analysed according to pre-defined descriptive variables that would facilitate accurate clinical referral: site, shape, size, skin/colour and presence of associated scars. To account for inter-regional variability, a pre-defined $95 \%$ overall descriptive variable inclusion rate standard was set. Size description sub-analysis was undertaken to study the descriptive accuracy of circular (Fig. 1) and oval (Fig. 2) lesions. The data were analysed using SPSS ver. 20 (IBM Corp., Armonk, NY, USA) and chi-squared tests undertaken as appropriate.

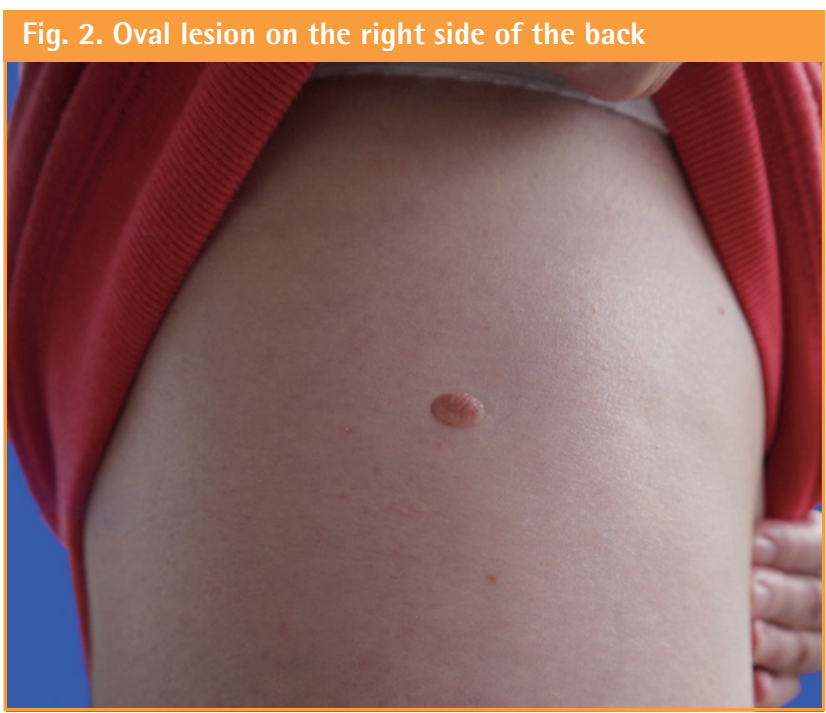




\section{RESULTS}

There were 250 lesion descriptions provided by 125 participants (response rate $=63 \%, 125 / 200)$ : consultants $(n=31)$, STs $(n=31)$, GPs $(n=30)$ and MSs $(n=33)$. Descriptive variable inclusion accuracy rates increased from MSs $(46 \%, 151 / 330)$ to GPs $(57 \%, 172 / 300)$, followed by STs $(68 \%, 211 / 310)$ and consultants $(80 \%, 249 / 310)$ (Table 1, Fig. 3). Descriptive variable inclusion rates were greater for consultants than for STs $(\mathrm{P}<0.001)$, GPs $(\mathrm{P}<0.0001)$ and MSs $(\mathrm{P}<0.0001)$, for STs than for GPs $(\mathrm{P}<0.01)$ and MSs $(\mathrm{P}<0.0001)$ and for GPs than for MSs $(\mathrm{P}<0.01)$. Descriptive variable sub-analysis by grade is outlined in Table 1. With the exclusion of mentioning absent associated scarring, descriptive variable inclusion rates increased from MSs (56\%, 148/264), to GPs (67\%, 161/240), STs (76\%, $189 / 248)$ and finally, consultants $(87 \%, 215 / 248)$ (Table 1).

Further study of size description pertaining to circular and oval dimensions (Fig. 4) indicated greatest accuracy for Consultants $(94 \%, 45 / 48)$, who outperformed STs $(73 \%, 32 / 44)$ $(\mathrm{P}<0.01)$ and MSs $(37 \%, 17 / 46)(\mathrm{P}<0.0001)$. STs and GPs $(80 \%, 24 / 30)$ both described circular and oval dimensions with greater accuracy than MSs $(\mathrm{P}<0.001)$. Of interest, in cases where inaccurate dimension descriptions occurred, the most common error was implication of a quadrilateral shape (66\%, $33 / 50)$, rather than a circle or oval, by consultants $(67 \%, 2 / 3)$, GPs $(83 \%, 5 / 6)$, STs $(67 \%, 8 / 12)$ and MSs $(62 \%, 18 / 29)$.

Table 1. Subanalysis of descriptive variables (\%) by grade and overall descriptive variable inclusion rates (\%) by grade, with and without the variable of mentioning absent associated scarring

\begin{tabular}{|c|c|c|c|c|c|c|c|}
\hline Characteristic & Site & Shape & Size & Skin/Colour & Scars & Overall & Overall (-) scars \\
\hline Consultants & $85(53 / 62)$ & $92(57 / 62)$ & $82(51 / 62)$ & $87(54 / 62)$ & $55(34 / 62)$ & $80(249 / 310)$ & $87(215 / 248)$ \\
\hline Speciality trainees & $76(47 / 62)$ & $68(42 / 62)$ & $71(44 / 62)$ & $90(56 / 62)$ & $36(22 / 62)$ & $68(211 / 310)$ & $76(189 / 248)$ \\
\hline General practitioners & $63(38 / 60)$ & $62(37 / 60)$ & $60(36 / 60)$ & $83(50 / 60)$ & $18(11 / 60)$ & $57(172 / 300)$ & $67(161 / 240)$ \\
\hline Medical students & $53(35 / 66)$ & $52(34 / 66)$ & $70(46 / 66)$ & $50(33 / 66)$ & $5(3 / 66)$ & $46(151 / 330)$ & $56(148 / 264)$ \\
\hline
\end{tabular}

\section{Fig. 3. Descriptive variable inclusion rates $(\%)$ by grade}

Descriptive variable inclusion rates increased from MSs (46\%, 151) $330)$ to GPs $(57 \%, 172 / 300)$, followed by STs $(68 \%, 211 / 310)$ and consultants $(80 \%, 249 / 310)$. The descriptive variable inclusion rates were significantly greater for consultants over STs $(P<0.001)$, GPs $(P<0.0001)$ and MSs $(P<0.0001)$, for STs over GPs $(P<0.01)$ and students $(P<0.0001)$ and for GPs over students $(P<0.01)$. MS, medical students; GP, general practitioners; $S T$, speciality trainees. ${ }^{*} \mathrm{P}<$ 0.05 , significant; ${ }^{* *} \mathrm{P}<0.01$, very significant; ${ }^{* *} \mathrm{P}<0.001$, highly significant; ${ }^{* * *} P<0.0001$, extremely significant.

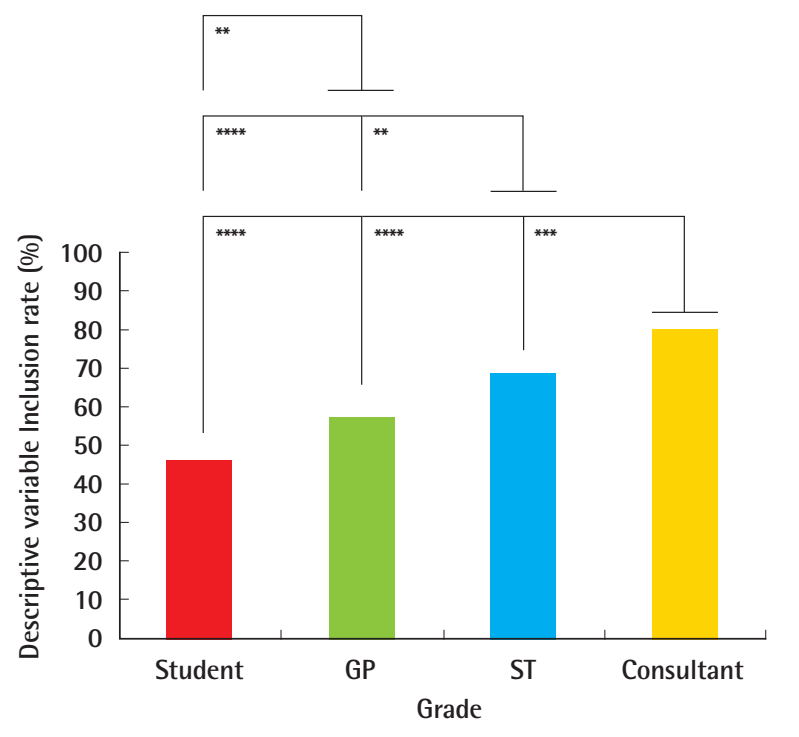

Fig. 4. Circular and oval dimension description accuracy (\%) by grade

Further study of size description pertaining to circular and oval dimensions indicated greatest accuracy for consultants $(94 \%, 45 / 48)$, who outperformed STs $(73 \%, 32 / 44)(P<0.01)$ and MSs $(37 \%, 17 / 46)$ $(\mathrm{P}<0.0001)$. STs and GPs $(80 \%, 24 / 30)$ both described circular and oval dimensions with greater accuracy than MSs $(P<0.001)$. ST, speciality trainees; GP, general practitioners; $M S$, medical students. ${ }^{*} P<$ 0.05 , significant; ${ }^{* *} \mathrm{P}<0.01$, very significant; ${ }^{* * *} \mathrm{P}<0.001$, highly significant; ${ }^{* * * P}<0.0001$, extremely significant.

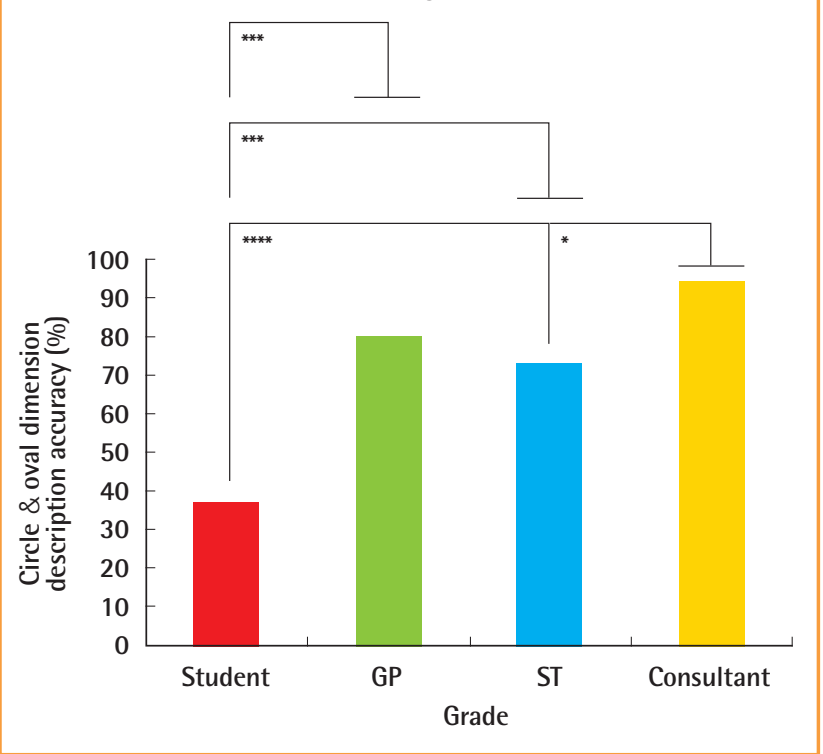




\section{DISCUSSION}

Descriptive variable inclusion rates did not meet the pre-defined audit standard of $95 \%$. However, neither lesion (Figs. 1, 2) was associated with scarring, so it could be argued that exclusion of this descriptive variable was legitimate. Although exclusion would increase the descriptive variable inclusion rates for all groups (Table 1), they would still not meet the standard. The authors, however, believe that explicitly noting an absence of associated scarring is 'an important negative', as it eliminates the possibility of post-surgical recurrence. The demonstrated increase in overall descriptive inclusion variable rates between groups (Fig. 3) reflects different specialist training exposure. MSs would have received the least training on skin lesions, and GPs, although adept at a broad spectrum of medical and surgical fields, are less likely to have experienced the same specialist training as STs, who in our centre may see 20 to 30 cases per half day outpatient clinic. Finally, consultants would have experienced the greatest specialist training exposure to such lesions, hence outperforming the other groups.

We have also demonstrated increased accuracy of circular and oval lesion dimension description with more training and experience (Fig. 4). In cases where inaccuracy occurred, the impression of a quadrilateral lesion was given; for example, the circular lesion (Fig. 1) may have been described as ' $4 \mathrm{~mm} \times 4 \mathrm{~mm}$ ' (i.e., a square), rather than as having a '4-mm diameter'. Similarly, the oval lesion (Fig. 2) may have been described as a ' $6 \mathrm{~mm} \times 4 \mathrm{~mm}$ lesion' (a rectangle), rather than as an oval lesion with 2 axial lengths. Square and rectangular lesions are unlikely to be encountered in clinical practice, and this highlights a level of descriptive inaccuracy that should be eliminated from the referral and management process.

These findings have clinical and practical implications, providing empirical evidence on the current level of lesion description performance from a national population sample and evidence of the positive effect of, and need for increased training [15]. Accurate skin lesion referral descriptions facilitate skin cancer services in identifying those that are longstanding and benign, rather than new and malignant, particularly when multiple, on large areas of sun-damaged skin or when affecting a poor historian. More accurate descriptions in ambiguous cases could spare patients from treatment producing unnecessarily large scars or defects requiring formal reconstruction, and the literature highlights a requirement for further studies on education $[14,15]$. Effort is required to ensure skin lesions are described as accurately as possible ('a circle is not a square') and we provide supporting evidence that speciality training and education improves this process [14]. BAD guidelines highlight the importance of careful lesion examination and diagnosis; we propose that greater focus be placed on learning these skills early in medical training, and maintaining them throughout clinical practice $[5,7,8]$.

\section{REFERENCES}

1. Cancer Research UK. Cancer incidence for all cancers combined [Internet]. London: Cancer Research UK; 2014 [cited 2014 Jul 20]. Available from: http://www.cancerresearchuk.org/cancer-info/cancerstats/incidence/all-cancers-combined/.

2. Cancer Research UK. Skin cancer statistic [Internet]. London: Cancer Research UK; 2014 [cited 2014 Jul 20]. Available from: http://www.cancerresearchuk.org/cancer-info/ cancerstats/types/skin/?script=true.

3. Department of Health. The NHS cancer plan [Internet]. London: Crown; 2000 [cited 2014 Jul 20]. Available from: http://www.thh.nhs.uk/documents/_Departments/Cancer/NHSCancerPlan.pdf.

4. Department of Health. The new NHS: modern, dependable [Internet]. London: Crown; 1997 [cited 2014 Jul 20]. Available from: http://webarchive.nationalarchives.gov. uk/+/www.dh.gov.uk/en/Publicationsandstatistics/Publications/PublicationsPolicyAndGuidance/DH_4008869.

5. The British Association of Dermatologists. Dermatology in the undergraduate medical curriculum [Internet]. London: The British Association of Dermatologists; 2006 [cited 2014 Jul 20]. Available from: http://www.bad.org.uk/library-media/documents/(Link2)\%20Core\%20curriculum(2).pdf.

6. Clayton R, Perera R, Burge S. Defining the dermatological content of the undergraduate medical curriculum: a modified Delphi study. Br J Dermatol 2006;155:137-44.

7. Schofield JK, Fleming D, Grindlay D, et al. Skin conditions are the commonest new reason people present to general practitioners in England and Wales. Br J Dermatol 2011; 165:1044-50.

8. Burge S, British Association of University Teachers of Dermatology. Teaching dermatology to medical students: a survey of current practice in the U.K. Br J Dermatol 2002; 146:295-303.

9. Cox NH, Madan V, Sanders T. The U.K. skin cancer 'twoweek rule' proforma: assessment of potential modifications to improve referral accuracy. Br J Dermatol 2008;158:1293-8.

10. Miranda BH, Soldin MS. Lumps 'n' bumps. In: Miranda BH, Asaad K, Butler PE, editors. DrExam part B MRCS OSCE revision guide book 2: clinical examination, communication skills \& history taking. Oxfordshire: Libri; 2010. p.3-14. 
11. Friedman RJ, Rigel DS, Kopf AW. Early detection of malignant melanoma: the role of physician examination and selfexamination of the skin. CA Cancer J Clin 1985;35:130-51.

12. Abbasi NR, Shaw HM, Rigel DS, et al. Early diagnosis of cutaneous melanoma: revisiting the ABCD criteria. JAMA 2004;292:2771-6.

13. Ashton RE. Teaching non-dermatologists to examine the skin: a review of the literature and some recommendations. Br J Dermatol 1995; 132:221-5.
14. Shariff Z, Roshan A, Williams AM, et al. 2-Week wait referrals in suspected skin cancer: does an instructional module for general practitioners improve diagnostic accuracy? Surgeon 2010;8:247-51.

15. Aldridge RB, Maxwell SS, Rees JL. Dermatology undergraduate skin cancer training: a disconnect between recommendations, clinical exposure and competence. BMC Med Educ 2012;12:27. 